\title{
INVESTIGACIONES
}

\section{EL MODELO DIALOGICO DE LA PEDAGOGIA: UN APORTE DESDE LAS EXPERIENCIAS DE COMUNIDADES DE APRENDIZAJE}

\author{
The dialogic educational model: a contribution based \\ on the learning communities experience
}

\author{
Donatila Ferrada ${ }^{1}$, Ramón Flecha ${ }^{2}$ \\ ${ }^{1}$ Universidad Católica de la Ssma. Concepción, Alonso de Ribera 2850, Concepción, Chile. \\ dferrada@ucsc.cl. \\ ${ }^{2}$ Universidad de Barcelona, Concilio de Trento 227, 5을 2 , Barcelona, España. \\ ramon.flecha@ub.edu
}

\section{Resumen}

En el transcurso de las dos últimas décadas en distintos contextos (España, Brasil, Chile) han emergido proyectos de transformación de los centros educativos -en escuelas que atienden alumnado en vulnerabilidad social y económica-, conocidos como Comunidades de Aprendizaje, cuya característica primordial es el involucramiento de toda la comunidad para conseguir aprendizajes de calidad en todo su alumnado. En la revisión de la abundante documentación publicada a la fecha que recoge todos estos proyectos de transformación de la escuela se descubre un nuevo modelo pedagógico implícito que, sin embargo, todavía no está sistematizado como tal en la bibliografía hoy existente. Este artículo realiza esa sistematización mediante el uso instrumental de categorías teóricas especializadas (concepto de educación, tipo de construcción de la persona, tipo de sociedad, enfoque curricular, modelo de aprendizaje, modelo didáctico y concepto de evaluación) y propone la emergencia de un nuevo modelo pedagógico que denominamos modelo dialógico de la pedagogía. De esta forma se intenta contribuir al campo específico de la pedagogía, en la sistematización de una alternativa pedagógica que resuelve

\begin{abstract}
Over the last two decades a transformational project has emerged in different contexts (Spain, Brazil and Chile) in schools that are mostly attended by pupils from vulnerable social and economic backgrounds. This project is entitled Learning Communities. The fundamental characteristic of this project is the involvement of the whole community in order to achieve a quality education for every pupil. When examining the abundant documents that have been published to date about this project, a new educational model comes to light. However, this model is still not systematised as such in the existing bibliography. This article will carry out that systematisation through the instrumental use of specialised theoretical categories (educational conceptualization, type of personal construction, type of society, focus of the curriculum, educational model, didactic model and concept of evaluation) and will propose a new educational model which has been entitled the dialogic educational model. In this way an attempt is being made in this article to contribute to the specific field of education, by systematising an educational alternative which efficiently resolves problems
\end{abstract}


efectivamente los problemas de fracaso, absentismo y conflicto escolar.

Palabras clave: comunidad de aprendizaje, currículum dialógico, aprendizaje dialógico, didáctica dialógica, evaluación dialógica. such school failure, absenteism and school conflicts.

Key words: learning communities, dialogic curriculum, dialogic learning, dialogic didactic, dialogic evaluation.

\section{COMUNIDADES DE APRENDIZAJE: UNA NUEVA FORMA DE HACER PEDAGOGIA}

El proyecto de Comunidades de Aprendizaje cuenta con una larga trayectoria de transformación social y educativa y desarrollo de una nueva pedagogía. El primer referente lo encontramos en la Escuela de Personas Adultas de La Verneda-Sant Martí (Sánchez, 1999), en un barrio obrero de Barcelona. Esta escuela se creó en el año 1978 como resultado de las reivindicaciones de los vecinos y las vecinas de la zona y, tras años de éxito en la superación de desigualdades formativas a través del aprendizaje dialógico, y su reconocimiento internacional, su modelo dialógico de pedagogía entró en práctica en la educación obligatoria. La primera comunidad de aprendizaje en educación primaria empezó en el País Vasco en el 1995. Tras su éxito en el aumento del aprendizaje de niños y niñas afectados por el fracaso escolar, así como la mejora de la convivencia, el proyecto se extendió a otras escuelas del País Vasco, recibiendo al poco tiempo apoyo del gobierno regional. Posteriormente, otras escuelas e institutos decidieron iniciar el proceso de transformación en comunidad de aprendizaje, siendo actualmente un total de 52 centros educativos en cuatro comunidades autónomas (País Vasco, Cataluña, Aragón, Castilla y Andalucía), los que funcionan como comunidades de aprendizaje en España, desde la educación infantil de primer ciclo al bachillerato. El proyecto, además, cuenta en la actualidad con gran apoyo institucional.

El éxito y consolidación actual de comunidades de aprendizaje en España en la superación del fracaso escolar y la mejora de la convivencia ha proyectado la experiencia a otros contextos, encontrando también ahora cuatro comunidades de aprendizaje en Brasil y dos en Chile.

El proyecto de transformación fundamenta sus acciones en la investigación. El Centro Especial de Investigación en Teorías y Prácticas Superadoras de Desigualdades (CREA) de la Universidad de Barcelona desarrolló el proyecto de reforma de centros educativos en comunidades de aprendizaje resultado de una larga trayectoria de investigación en las mejores teorías y prácticas internacionales para la superación del fracaso escolar y el aumento de la cohesión social. Todas estas teorías y prácticas coinciden en señalar que la clave del aprendizaje en la sociedad de la información está en la interacción comunicativa y en la coordinación de los diferentes contextos educativos. Fruto de estas investigaciones, CREA ha asesorado a los centros educativos que han decidido iniciar el proyecto de transformación educativa y sociocultural en comunidad de aprendizaje, coordinando un trabajo de colaboración con escuelas e institutos que atienden mayoritariamente alumnado que sufre desigualdades (principalmente sectores sociales excluidos del desarrollo de España, tales como gitanos, 
inmigrantes latinoamericanos y africanos, y españoles en situación de vulnerabilidad socioeconómica). En términos generales, una comunidad de aprendizaje se define como "un proyecto de transformación social y cultural de un centro educativo y de su entorno, para conseguir una sociedad de la información para todas las personas, basada en el aprendizaje dialógico, mediante educación participativa de la comunidad que se concreta en todos sus espacios, incluida el aula" (Elboj y otros 2002). Desde este mismo referente, en Brasil y en Chile se inicia la transformación de escuelas en comunidades de aprendizaje, considerando las particularidades propias de cada contexto social, cultural y político.

Todos estos centros educativos comparten un modelo dialógico de pedagogía que gira en torno a una nueva concepción de la educación, el currículum, el aprendizaje, la didáctica y la evaluación. Así también comparten resultados altamente exitosos -guardando las diferencias asociadas a su extensión en el tiempo-, cuya clave de lo mismo ha sido la asunción paradigmática y programática del modelo dialógico, en el trabajo pedagógico que realiza la escuela.

Esta concepción comunicativa de la pedagogía supera antiguas concepciones pedagógicas de la sociedad industrial, como el aprendizaje tradicional de la concepción objetivista y el aprendizaje significativo de la concepción constructivista. Desde este modelo dialógico de pedagogía, las comunidades de aprendizaje están haciendo frente con éxito a las consecuencias derivadas de la primera fase de la sociedad de la información, donde el énfasis en la idea de diferencia sin considerar la igualdad aumentó las desigualdades educativas y sociales.

Sobre la base de estos antecedentes, el levantamiento de este nuevo modelo pedagógico obedece a una revisión actualizada de la pedagogía, cuyo objeto de estudio comprende toda intencionalidad de educabilidad humana bajo espacios formales e informales de la institución educativa y las distintas dimensiones y agentes que interactúan con ella (Meneses 1999; Hoyos 1999). Desde esta comprensión, la pedagogía organiza su propia praxis desde dos planos fuertemente interrelacionados. El primer plano eminentemente teórico, ofrece respuestas a las siguientes preguntas: 1) ¿qué noción de educación orientará la acción pedagógica?, lo que se traduce en la explicitación del concepto de educación que define la acción; 2) ¿qué tipo de persona se desea formar?, que implica evidenciar cómo se construye la persona desde sus distintas dimensiones, las cuales habría que considerar en el proceso de formación; y 3) ¿qué tipo de sociedad se quiere construir?, lo que se traduce en asumir una explicación del funcionamiento de la sociedad en correspondencia con las dos preguntas anteriores. El segundo plano es operativo y ofrece respuesta a las siguientes preguntas en plena correspondencia con el plano anterior: 1) ¿qué enseñar?, lo que implica explicitar un concepto de currículum y una selección de conocimientos educativos que se transforman en el currículum a desarrollar en las instancias pedagógicas formales e informales; 2) ¿cómo aprenden las personas a las que se quiere enseñar?, lo que implica asumir un modelo de aprendizaje; 3) ¿cómo enseñar?, lo que implica asumir un modelo didáctico; y 4) ¿cómo evaluar los aprendizajes alcanzados?, lo que implica, al menos explicitar un concepto de evaluación. 


\section{CONSIDERACIONES METODOLOGICAS}

La metodología usada en el levantamiento del nuevo modelo pedagógico es la concepción comunicativa crítica (Gómez y otros 2006), puesto que permite la identificación de dimensiones transformadoras respecto de dimensiones exclusoras en los procesos de construcción de conocimiento. En correspondencia, se usó la técnica comunicativa de análisis de contenidos de textos, mediante el uso instrumental de las siguientes categorías teóricas especializadas, con lo cual se identificó tanto la dimensión transformadora como la exclusora: 1) concepto de educación (Eisner 2002a; Bernstein 1993; Lundgren 1992); 2) tipo de construcción de persona (Lundgren 1992; Soto 1996; Eisner y Wallance 1974); 3) tipo de sociedad (Bernstein 1985; Eisner 1998, Lundgren 1992); 4) enfoque curricular (Eisner y Wallance 1974; Bernstein 1993; Lundgren 1992; Grundy 1991), a través de sus subcategorías: concepto de currículum y selección del conocimiento educativo; 5) modelo de aprendizaje (Flecha 2000; Racionero y Valls 2007; Aubert y Soler 2007; Ferrada 2007), a través de la expresión de sus ocho principios: diálogo igualitario, inteligencia cultural, construcción de sentido, dimensión instrumental, transformación, solidaridad, igualdad de diferencia, emocionalidad; 6) modelo didáctico del trabajo en el aula (Quintanilla y otros 2005; Chevalard 1991; Molero 1999; Labarrere y Quintanilla 2002; Pozo y Gómez 2003; Porlán y Rivero 1998), a través de los elementos reguladores de su acción: metodología, rol de profesor, rol del alumnado, organización del aula; 7) concepto de evaluación (Eisner, 2002b).

Las unidades de textos se seleccionaron en función de su validación en la comunidad científica internacional (Comité editorial activo y revistas indexadas ISI y Scielo), quedando constituidas en un total de 21 textos (4 libros ${ }^{1}$ : L1, L2, L3, L4; 6 artículos $^{2}:$ A1, A2, A3, A4, A5, A6; 4 tesis doctorales 3 : TD1, TD2, TD3, TD4; 4 tesis

$1 \quad$ L1: Elboj, C.; Puigdellívol, I.; Soler, M.; Valls, R. (2002). Comunidades de Aprendizaje. Transformar la Educación. Barcelona, Graó. L2: Flecha, R. (1997). Compartiendo Palabras. El aprendizaje de las personas adultas a través del diálogo. Barcelona, Paidós. L3: Aubert, A.; Duque, E.; Fisas, M.; Valls, R. (2004). Dialogar y transformar. Pedagogía crítica del siglo XXI. Barcelona, Graó. L4: Ferrada, D. (2001). Currículum Crítico Comunicativo. Barcelona, El Roure.

2 A1: Racionero, S.; Valls, R. (2007). Dialogic Learning: A communicative approach to teaching and learning. The Praeger Handbook of Educational and Psychology Vol. (3), Chapter 65: 548-557. A3; A2: Aubert, A.; Soler, M. (2007). Dialogism: The diagotic turn in the social sciences. The Praeger Handbook of Educational and Psychology Vol. (3), Chapter 62: 521-529. A3: Sánchez, M. (1999). La Verneda-Sant Martí: A School where people dare to dream. Harvard Educational Review. Vol. 69, Nº 3: 2-24. A4: Flecha, R. (1996). Out of the ghetto - a communicative perspective. International Journal of University Adult Education. United Kingdom: University of Warwick, No 3, v. 35: 1-7. A5: Flecha, R. \& Puigvert, L. (2005). Formación del profesorado en las comunidades de aprendizaje. Revista Colombiana de Educación, Núm. 48, Bogotá: Universidad Pedagógica Nacional; A6: Ferrada, D. (2008). El principio de emocionalidad: Un aporte al modelo dialógico del aprendizaje. Revista REXE: Vol. 7 No 14: 32-46.

3 TD1: García, C. (2004). Comunidades de Aprendizaje. De la Segregación a la Inclusión. Tesis para optar al grado de Doctora en Sociología. Universidad de Barcelona. TD2: Elboj, C. (2001). Comunidades de Aprendizaje. Un modelo antirracista en la sociedad de la información. Tesis para optar al grado de Doctora en Sociología. Universidad de Barcelona. TD3: Diez, J. (2004). La enseñanza de las matemáticas en la educación de personas adultas. Un modelo dialógico. Tesis para optar al grado de Doctor en Didáctica de las Matemáticas. Universidad de Barcelona. TD4: Valls, R. (2000). Comunidades de Aprendizaje. Una práctica educativa de aprendizaje dialógico para la sociedad de la información. Tesis para optar al grado de Doctora en Sociología. Universidad de Barcelona. 
de Magíster4: TM1, TM2, TM3, TM4; 1 Tesis de Licenciatura en Educación 5: TL1; y 2 Informes de investigación 6 : I1, I2). Por su parte, la validación de la data se realizó de acuerdo a los criterios de rigor de la metodología comunicativo crítica (Diálogo intersubjetivo, Pretensiones de validez y compromiso).

\section{EL MODELO DIALOGICO DE LA PEDAGOGIA}

La construcción del modelo dialógico de la pedagogía se presenta en función de los resultados encontrados. Primero, desde la construcción de cada categoría definida producto del análisis de contenido de texto, seguidamente de la identificación de la dimensión transformadora respecto de la dimensión exclusora, para finalmente sistematizar cada una de ellas.

1) CONCEPTO DE EDUCACION. Se comprende como un medio para promover interacciones humanas dirigidas a transformar las propias construcciones intersubjetivas de quienes participan en el acto educativo al interior de una comunidad con predominio de la racionalidad comunicativa. Esto fundamenta que quienes participan adquieren protagonismo para intervenir en cada una de las decisiones educativas, mediante las interacciones que mantienen entre sí para la construcción de nuevos significados cuando deciden emprender proyectos conjuntos dirigidos a transformar la escuela. Desde esta comprensión, educar significa, precisamente la transformación de cada sujeto que enseña y aprende resultado de sus múltiples interacciones solidarias con los demás, donde sus acciones y opciones son válidas y tienen cabida en la escuela, si son justificadas desde pretensiones de validez. Este concepto de educación dinamiza desde el propio sistema de creencias que porta el profesorado y todos los implicados en un proceso educativo, al mismo tiempo que orienta cada una de las acciones prácticas que se realizan cotidianamente en los distintos espacios de la escuela (L1; L2; L3; L4; A5; A6; TM1; TM2; TM3; TM4).

4 TM1: Colipí, N. (2008). "La igualdad de oportunidades de aprendizaje por medio del aprendizaje dialógico en la escuela pública chilena”. Tesis para optar al grado de Magíster. Universidad Católica de Concepción. Chile TM2: Melo, C. (2008). "Diversidad de interacciones sociales en los alumnos como medio de generación de aprendizaje de tipo instrumental y social”. Tesis para optar al grado de Magíster. Universidad Católica de Concepción. Chile. TM3: Saldaña, D. (2008). El rol de los colaboradores de aprendizajes al interior del proyecto Enlazando Mundos. Tesis para optar al grado de Magíster. Universidad Católica de Concepción, Chile.TM4: Bastías, E. (2008). "La Gestión Educativa Construida al Interior del Proyecto Educativo Enlazando Mundos. Modelo de gestión y Conceptos de participación”. Tesis para optar al grado de Magíster en Ciencias de la Educación. Universidad Católica de Concepción. Chile.

5 TL1: Andaur, A.; Screiber, B.; González, M.; Venegas, C. (2008). "El desarrollo profesional docente en el contexto del proyecto "Enlazando Mundos": Una redefinición del rol profesional del profesorado en dos escuelas públicas de la comuna de Concepción". Tesis para optar al grado de Licenciado en Educación. Universidad Católica de Concepción. Chile.

6 I1: Soler, M. (2003). Lectura dialógica e igualdad de género en las interacciones del aula. Memoria final de Investigación. Ministerio del Trabajo y Asuntos Sociales. Instituto de la mujer. I2: Valls, R. (2005). Prevención de la violencia de género en el contexto educativo a partir de nuevas formas de organización escolar. Proyecto de la Generalitat de Catalunya. Institut Català de les Dones. Universidad de Barcelona. 
De esta forma, la dimensión transformadora del concepto de educación se sitúa en la comprensión -por parte de todos quienes participan en ella- de que es posible cambiar las propias construcciones intersubjetivas que se portan. Por el contrario, la dimensión exclusora del concepto de educación la concibe como una estructura, un conjunto de prácticas orientadas a preservar y reproducir conocimiento, costumbres y tradiciones de la sociedad, manteniendo así la estructura social. La función de la educación es simplemente la reproducción y no la producción de conocimiento, por lo tanto, referida al resultado de una acción donde el alumno es el producto. En esta perspectiva el educar significa enseñar a conocer lo que no se sabe, es un interés por la supervivencia mediante el control y la manipulación del ambiente. Así la dimensión exclusora implica situarse en la imposibilidad de movilizar cambios e inmovilizar con ello cualquier intento de cambio.

En consecuencia, el concepto de educación que orienta el modelo dialógico de la pedagogía implica una praxis de transformación desde las propias construcciones intersubjetivas de quienes comprenden la educación como un proceso que les involucra protagónicamente, y que no es sólo responsabilidad de la comunidad educativa, sino de toda la comunidad, hasta las acciones más cotidianas del trabajo pedagógico en el aula y fuera de ella donde toda la comunidad participa colaborando solidariamente.

2) TIPO DE CONSTRUCCION DE PERSONA. Desde esta construcción se asume que la persona humana está dotada de la acción, por tanto es agente-actuante, constructora y transformadora del medio en el cual se desarrolla y vive. Se sostiene que la formación de la persona cuenta con dos mecanismos de regulación que la constituyen como unidad. El primero, es el centro autopoiético, que constituye en sí y como totalidad orgánica el propio mecanismo regulador, que la hace humana en la interacción con el entorno emotivo y amoroso - mediatizado por el fluir del lenguaje- modificando así su propia fisiología a la vez que modifica el lenguaje. El segundo, es el centro intersubjetivo, que se regula desde su lenguaje y acción, dotándola de actitud crítica resultado de las interacciones que mantiene con sus personas de referencia, que a su vez la dotan de responsabilidad en sus actos y en el uso del lenguaje. La conexión entre uno y otro centro regulador está dada por el lenguaje. Este planteamiento, que sostiene la construcción de la persona en un núcleo intersubjetivo-autopoiético, sirve de sustento para la conducción de un proceso transformador que requiere de la persona y de su propio colectivo en el agenciamiento de sus propias intenciones educativas y sociales. Desde esta visión, la pregunta de la pedagogía por el tipo de construcción de persona encuentra su base en el desarrollo y fortalecimiento de las interacciones humanas, las cuales, cuanto más diversas y heterogéneas, más posibilidades de transformación de las propias construcciones intersubjetivas (L4; A6).

Desde la dimensión transformadora, es posible identificar el protagonismo de la persona tanto en la construcción de sí misma (siempre frente a los ojos de y en interacción con los demás), como también en la sociedad en que vive, fundamento que permite echar por tierra la dimensión exclusora, de la creencia de que la persona es un ser eminentemente pasivo desde un punto de vista social y que se construye casi exclusivamente por procesos internos ya sean naturales (inherentes a ella) o psicológicos (principalmente 
dirigidos a la adaptación). Estos últimos constituyen los principales fundamentos utilizados por visiones más tradicionales de la pedagogía que sostienen una inamovilidad en la construcción de la persona, la cual la deja fuera de todo protagonismo en cualquier decisión escolar y educativa.

En consecuencia, el tipo de construcción de la persona conceptualiza una nueva dimensión de praxis humana, ahora con una doble dimensión, biológica y social que la dota de iniciativa y protagonismo, tanto en su propia construcción como persona como frente a la sociedad a la que pertenece, al mismo tiempo que refuta que la persona humana sea un ser naturalmente pasivo e incapaz de comprometerse en ningún cambio social. Así, la pregunta de la pedagogía sobre qué tipo de persona formar responde a esta doble dimensión de praxis, incorporándola, junto a otros agentes sociales, en las decisiones respecto de qué se persigue formar.

3) TIPO DE SOCIEDAD. El análisis de contenido de textos realizado en este estudio evidencia una clara coincidencia en la caracterización de la sociedad como una sociedad dialógica, denominada así porque el diálogo asume un protagonismo mucho mayor que en la sociedad industrial y que en las sociedades premodernas, aspecto que permite abrir nuevas formas de convivencia, de conversación y reflexión conjunta entre grupos humanos diferentes (de culturas, de géneros, de generaciones, de etnias, de organización familiar, entre muchos otros) que persiguen y claman ser incluidos en la sociedad respetando su diferencia en igualdad de condiciones. En esta dimensión, es el diálogo el que se transforma en la bandera de lucha de la mayoría de los grupos y no la fuerza impositiva del poder o la violencia de las armas para alcanzar la inclusión. En este mismo marco, se asume la perspectiva habermasiana de la sociedad cuyo funcionamiento es doble, como sistema y como mundo de la vida. Entendiendo, al primero, como aquel entramado de interrelaciones entre los subsistemas económico y administrativo con los órdenes institucionales del mundo de la vida (esferas públicas y privadas) cuyos recursos (dinero y poder) controlan las funciones de rendimiento organizativo y las decisiones políticas (subsistema administrativo) y los bienes y servicios y fuerza del trabajo (subsistema económico). Como mundo de la vida simbólicamente estructurada, la sociedad es una comunidad en comunicación que se entiende en el horizonte del acervo cultural aproblematizado que constituye el sustrato común que comparten sus miembros y que desde ese horizonte entran en relaciones diversas. Desde esta afirmación, toda vez que las personas realizan procesos de interpretación contribuyen con algo a la cultura, a la vez que es la propia cultura la que está proporcionando los contenidos para ello, por tanto no se puede afirmar que es sólo el individuo quien la produce, ni que él se hace acreedor de la cultura como un receptáculo vacío, sino que ambos se retroalimentan mutuamente. Lo mismo ocurre a nivel de los procesos de socialización, en que sólo a partir de las operaciones de coordinación de las personas que actúan comunicativamente se forman redes de interacción de grupos más o menos integrados, de grupos que se mantienen de forma más o menos solidariamente, teniendo presente que las personas no pueden ser descritas como portadoras de estas redes de interacción, es decir, las interacciones no son algo que porten inherentemente, sino que son resultado de la retroalimentación entre los propios individuos y la sociedad a la que pertenecen. Estos procesos de socialización y formación construyen la personalidad de los individuos; ambos procesos son dependientes de los 
aprendizajes que desarrollan las propias personas inmersas en una cultura que ofrece contenidos posibles de interpretar y de dotar de nuevos significados dependientes del espectro de pretensiones de validez que se genere, hecho que también ocurre cuando están inmersos en una sociedad que funciona bajo órdenes normativos, en los que también puede cambiarse el horizonte lingüístico bajo nuevos contextos de pretensiones de validez, si viene el caso. Todas estas situaciones representan procesos de aprendizajes que van configurando la personalidad (L1; L2; L3; L4).

La escuela, al igual que la sociedad, en tanto orden institucional perteneciente al dominio de la esfera pública, también las funciones de transmisión de la cultura (fundamentalmente de la que ha sido seleccionada para tales efectos); integración social (de los estudiantes a la comunidad a la que pertenecen, a través del conjunto de normas y reglas que rigen en ella); y socialización (en cuanto a la formación de la personalidad de los estudiantes). Todas estas funciones pertenecen al ámbito del mundo de la vida, por lo que estamos en condiciones de afirmar que la escuela funciona como tal, y, por tanto, para llevar a cabo estas funciones sólo puede operar bajo contextos de la acción comunicativa. De la misma forma, la escuela funciona como sistema en tanto cuenta con un subsistema administrativo interno que a su vez depende de otro mayor, el cual lo dota de los recursos humanos (contratación de profesionales y personal de apoyo), materiales (infraestructura) y legales (reglamentos) que permiten su funcionamiento. Asimismo, la escuela debe responder a los estándares que le exige el propio sistema que regula mediante ellos, la propia inclusión de los individuos a la sociedad en general (L4). Todos estos aspectos se consideran en una comunidad de aprendizaje, puesto que aportan los elementos fundamentales para sostener el proceso de transformación que requiere la escuela (L1; L3; L4).

Desde estos resultados y frente a la pregunta de ¿qué tipo de sociedad se quiere construir?, la respuesta es una sola: consolidar una sociedad dialógica desde el funcionamiento del sistema y del mundo de la vida. Desde el mundo de la vida, junto con reproducir las estructuras simbólicas del mundo de la vida necesarias para la mantención de una cultura y una sociedad, sea al mismo tiempo capaz de incorporar procesos de renovación e innovación requeridos para el enriquecimiento de los mismos, como también de la apertura de espacios que permitan revertir los fenómenos de crisis (pérdida de sentido, pérdida de legitimación, desestabilización de las identidades colectivas, alienación, pérdida de motivación, etc.), con lo cual iniciar la transformación social. Desde el sistema, formar sujetos sociales altamente competentes en los saberes instrumentales requeridos para su inclusión en la sociedad.

Desde la dimensión transformadora, es posible evidenciar que el giro dialógico de la sociedad actual genera nuevos espacios para la superación de las desigualdades sociales y para avanzar efectivamente en una sociedad más inclusiva e igualitaria. En cambio, desde la dimensión exclusora, el anclaje al planteamiento en que se niega el valor del diálogo como mecanismo que regula la movilización de los procesos de transformación que persiguen los distintos sujetos inmoviliza cualquier acción transformadora.

En consecuencia, esta pedagogía explica el funcionamiento de la sociedad y la escuela desde la dimensión de dialogicidad, desde lo cual se requiere de una formación en aquellos saberes que sustentan la sociedad dialógica a fin de hacer conocedores de opciones teóricas que ofrecen posibilidades de transformación a todos los involucrados en los procesos de formación, y no sólo posturas teóricas fatalistas. Asimismo, el modelo dialógico de la pedagogía enfatiza la importancia de la acción educativa colectiva 
mediatizada por la comunicación como medio para combatir la colonización sistémica del mundo de la vida en las escuelas y las comunidades.

4) ENFOQUE CURRICULAR. Desde el marco de la definición de la pedagogía y en el plano operativo de la misma el enfoque curricular responde una de sus preguntas fundamentales: ¿qué enseñar en la escuela?, lo que implica tomar decisiones respecto de bajo ¿qué concepto de currículum? se decidirá ¿qué contenidos seleccionar de la inmensidad de contenidos existente en la actual sociedad del conocimiento?

4.1. El concepto de currículum se define como una construcción social que surge de las múltiples y diversas interacciones de las personas que componen una comunidad educativa contextualizada histórica y socialmente, y que están sujetas tanto a relaciones de poder, como también de diálogo. La pregunta por el qué enseñar queda resuelta por la propia comunidad que participa en esta construcción social, considerando la totalidad del contexto en que se toman las decisiones, es decir, tanto el local como el global (L4; A6; TM1; TM2; TM3; TM4).

La dimensión transformadora del concepto de currículum implica la asunción de unas prácticas educativas profundamente dinámicas y sobre la base de la participación de una diversidad de grupos heterogéneos que participan y toman decisiones sobre el qué enseñar en la escuela. Esto hace que la escuela considere una multiplicidad de variables que intervienen en el proceso educativo, donde los sujetos que participan son reconocidos en su heterogeneidad, al mismo tiempo que se conjugan todas esas variables para la generación de igualdad educativa. En cambio, desde la dimensión exclusora, el concepto de currículum viene a constituirse en una práctica planificada de acuerdo a objetivos predefinidos e inamovibles, sin participación de la propia comunidad educativa, puesto que el qué enseñar lo deciden sólo los especialistas. Estos contenidos controlan el ambiente educativo, de manera que el producto (estudiante) se ajuste a los objetivos predeterminados. Esto hace que la escuela tradicional parta del supuesto de que todos sus alumnos son iguales y se instalen prácticas curriculares homogéneas en una realidad profundamente heterogénea, con escasísimos resultados de igualdad educativa.

En consecuencia, el concepto de currículum, que orienta el qué enseñar, desde una dimensión dialógica de la pedagogía, implica la participación protagónica de todos los agentes sociales y educativos involucrados en los procesos curriculares de la escuela (expertos, profesores de aula, equipos directivos, alumnado, familiares y vecinos, entre otros), así como el cumplimiento de los objetivos curriculares para todo el alumnado, sin exclusiones.

4.2. Selección del conocimiento educativo. Sobre la base de las categorías anteriores, se construye que es esencial y fundamental el establecimiento de acuerdos sobre los propios criterios que serán usados por la comunidad que decide participar en las decisiones y acciones curriculares. De esta forma, los criterios se construyen colaborativamente entre todos los miembros involucrados en la transformación de la escuela, así son tan válidos los definidos como obligatorios por currículum nacional como los aportados por los otros participantes en la construcción colectiva del currículum (L4).

Desde esta comprensión, es posible evidenciar acuerdos sobre los dos ámbitos de la sociedad, por considerarse esenciales en todos los proyectos estudiados: el sistema, a saber, 
saberes sistematizados por la ciencia, la técnica y la tecnología que denomina saberes instrumentales (L2; L4; A2; A5) (planes y programas oficiales Objetivos Fundamentales Verticales, Contenidos Mínimos Obligatorios, los solicitados por el propio alumnado de la escuela, los propuestos por el profesor, y los propuestos por familiares y voluntarios que participen en la escuela); y el mundo de la vida, a saber, saberes provenientes de la cultura, la sociedad y la personalidad, que denomina saberes sociales (L2; L4; TM1; TM2; TM3; TM4; A6) (Objetivos Fundamentales Transversales definidos en los programas oficiales, los emanados del proyecto de centro, del contexto en que está ubicada la escuela, del contexto nacional, del contexto internacional, los contenidos emergentes desde el propio alumnado, los contenidos emergentes de los propios familiares y voluntarios) (L4; A6; TM1; TM2; TM3; TM4).

Desde una dimensión transformadora, es posible identificar la incorporación al currículum de nuevas relaciones de legitimidad y valoración de patrones normativos y conocimientos tradicionalmente excluidos, omitidos, rechazados y prohibidos de la escuela, además de los tradicionalmente incluidos, aceptados y permitidos, todos los cuales siempre deben poder ser enjuiciados por los propios miembros que participan en la comunidad educativa. Estos hechos permiten iniciar procesos de interpretación, significación y crítica de cada uno de estos contenidos. Por el contrario, desde la dimensión exclusora, es posible identificar el acuerdo entre profesionales de la educación de la rebaja de contenidos educativos en sectores sociales mayormente excluidos, lo cual ha traído como consecuencia un distanciamiento entre este alumnado y aquel que pertenece a sectores sociales incluidos en la sociedad actual.

En consecuencia, la selección de conocimiento educativo requiere de una construcción y validación colectiva, esto se constituye como la base para asumir el protagonismo colectivo necesario para transformar el fracaso en éxito escolar. Estos constituyen los fundamentos esenciales en que se asienta la participación del profesorado, el alumnado, los familiares, el vecindario y cualquier otro ciudadano que desee participar en la escuela, así como su capacidad para transformar las desigualdades de aprendizaje del alumnado en aprendizajes de máximos para todos. Para ello, y especialmente para aquellos colectivos en riesgo de exclusión, es esencial la priorización de los contenidos que son requeridos para la inclusión social y laboral, y la plena participación en la actual sociedad del conocimiento. El aprendizaje de estos contenidos debe ir acompañado de una reflexión crítica.

5) MODELO DE APRENDIZAJE. Los textos analizados exponen un modelo de aprendizaje asumido por todas las escuelas que se han transformado en Comunidades de Aprendizaje, que tiene como característica esencial la promoción y generación de una igualdad de aprendizaje en todo el alumnado, denominado aprendizaje dialógico, el cual se obtiene como resultado de la interacción dialógica entre todas las personas que participan en instancias educativas o que, sin participar, son claves para el aprendizaje de los y las estudiantes. Este modelo de aprendizaje se organiza en torno al siguiente conjunto de principios básicos (L2):

5.1) Diálogo igualitario: Se produce cuando todas las personas participantes (profesionales de la educación, familiares, administradores, participantes en asociaciones y entidades del barrio, estudiantes) tienen las mismas oportunidades para intervenir en 
los procesos de reflexión y de toma de decisiones sobre temas educativos relevantes. Las personas participan en un plano de igualdad, donde son importantes los aportes de cada uno de los integrantes, según los argumentos presentados, no según la mayoría o según una imposición de poder, derivada, por ejemplo, de la jerarquía dentro de la estructura escolar. Los participantes se han de acostumbrar a argumentar sus opiniones y a no recurrir a criterios de autoridad, tampoco se debe aceptar que cualquier cosa vale (L1; L2; L3; A2; A5; TD1).

La dimensión transformadora de este principio se identifica en la aceptación -por parte de todo el colectivo- de la argumentación como el eje central desde el cual se puede conducir un proceso de aprendizaje. En cambio, la dimensión exclusora se identifica en la imposición de temáticas educativas sobre la base de pretensiones de poder.

5.2) Inteligencia cultural: Es la capacidad de aprender a través del diálogo y de las habilidades comunicativas que tienen las personas. La inteligencia cultural se refiere a una capacidad universal, así todas las personas tienen inteligencia cultural porque tienen capacidades innatas para comunicarse y actuar gracias al lenguaje. La inteligencia cultural va más allá de la inteligencia académica, que sólo muestra que la persona ha pasado por la escuela y que vive rodeada de la cultura académica occidental, blanca y de clase media. Por el contrario, la inteligencia cultural significa el reconocimiento de la capacidad de aprendizaje y de la inteligencia de todas las personas, de cualquier cultura, barrio, etnia o edad. Si, por el contrario, aceptamos que no tienen esa capacidad, rebajaremos los niveles para adaptarlos, desde nuestra pretendida superioridad cultural. El aprendizaje dialógico requiere reconocer por igual los tres tipos de inteligencia (académica, práctica y comunicativa) y favorecer la transferencia de unas a otras (L1; L2; L3; A2; A5).

La dimensión transformadora, en este principio, se identifica en la búsqueda del desarrollo pleno de todas las inteligencias en todos los sujetos independiente desde cual partan éstos. En cambio, la dimensión exclusora, es la mantención de la inteligencia académica como la única expresión de inteligencia que valida la escuela.

5.3) Transformación: Para superar el fracaso escolar es necesario un cambio en el contexto sociocultural del cual provienen los niños y niñas, poner en práctica todos los medios para que cada persona llegue al máximo de sus posibilidades de aprendizaje, en superar el recurso al dictamen como etiquetaje paralizador y modificarlo en instrumento de transformación de una situación personal o social que no puede convertirse antes de los diez años -como muchas veces sucede- o a la edad que sea, en una marca que imposibilita toda capacidad de mejora de una persona (L1; L2; L3; A2; A5).

La dimensión transformadora, en este principio, es la generación de altas expectativas de todo el alumnado, sin distinción alguna, y la transformación del entorno sociocultural para promover los máximos aprendizajes. Por el contrario, la dimensión exclusora es la mantención de la distribución del alumnado de acuerdo a estereotipos sociales, cognitivos, étnicos, de género, etc., conduciendo a prácticas adaptadoras al contexto, cuando este presenta dificultades, en lugar de transformarlo.

5.4) Dimensión instrumental: Son los conocimientos científicos y técnicos y el desarrollo de habilidades instrumentales necesarias para actuar con éxito en la sociedad. La selección de estos aprendizajes provenientes del ámbito de la ciencia y de la técnica 
requiere de la participación del estudiantado y de sus familiares. Tradicionalmente, se ha excluido a estos agentes desde la escuela aludiendo a su falta de conocimientos, pero justamente la experiencia que poseen fuera del ámbito educativo les da un conocimiento plural y más real de lo que acontece en los extramuros de la escuela que es esencial para contextualizar el aprendizaje instrumental. Además, las familias y otros miembros de la comunidad no profesionales conocen el funcionamiento y las exigencias del mercado laboral y saben perfectamente qué van a necesitar sus hijos e hijas para estar incluidos en la sociedad del conocimiento. El alumnado, a su vez, tiene cada vez mayor acceso a fuentes de información que amplía sus intereses y necesidades formativas. El aprendizaje instrumental se intensifica cuando se desarrolla en un contexto de diálogo entre iguales, en el que participan diversidad de personas de la comunidad, puesto que permite tener en cuenta todas sus dimensiones de la inteligencia humana y favorecer los procesos de transferencia gracias a las habilidades comunicativas de todos y todas las estudiantes (L1; L2; L3; A2; A5).

La dimensión transformadora de este principio se evidencia al considerar que los saberes instrumentales, anteriormente definidos como propios del funcionamiento de la sociedad como sistema, debe alcanzarlos todo el alumnado, no sólo unos pocos. En cambio, la dimensión exclusora se muestra cuando se sostiene que esto no es tan necesario en determinados sectores sociales, en determinadas etnias, en determinados grupos con limitaciones cognitivas, etc., reproduciendo así las desigualdades que esos grupos ya sufren.

5.5) Creación de sentido: Vivimos en una sociedad abierta a una pluralidad creciente de estilos de vida, la posibilidad de elegir el proyecto vital requiere de un tener acceso a un abanico de posibilidades que puede ofrecer la educación, presentando la posibilidad de compartir y de discutir sobre diferentes estilos de vida. Así, los niños y niñas que conocen -en su medio social- un solo tipo de trabajo, que es el de sus familias, por ejemplo, peluqueras o vendedoras, muchas veces ocurre que sus aspiraciones se restringen a reproducir esos mismos empleos, el conocer otras posibilidades y el confiar en la educación para lograrlo implica la construcción de sentido para este alumnado, puesto que puede, ahora, elegir libremente (L1; L2; L3; A2; A5).

La dimensión transformadora de este principio se visualiza en la posibilidad real de mostrar alternativas de proyectos de vida para alumnado que en sus experiencias cotidianas tiene acceso a escasa diversidad de éstos, y crear las condiciones para que esos proyectos se hagan realidad. De esta forma se acortan las distancias entre lo que se desea y espera de la escuela y la experiencia cotidiana en el centro, lo que es fuente de sentido para toda la comunidad. En cambio, la dimensión exclusora se observa cuando se mantiene la restricción de proyectos de vida diversos.

5.6) Solidaridad: Es un valor que se consigue sólo a través de la práctica solidaria, como un valor que se pone en práctica en las interacciones que desarrollamos en el aula y, en primer lugar, por parte de las profesoras y profesores. La solidaridad se da cuando se genera la convivencia multicultural, multisocial, multiprofesional, no cuando se presenta como algo que se debe evitar. Solidaridad también es compartir procesos de aprendizaje en vez de separar de forma competitiva al alumnado que va a desarrollar conocimientos y herramientas más relevantes por estar más capacitado, más interesado o más motivado, 
de otros estudiantes que se colocan en grupos de muy bajo nivel y que coinciden pertenecer a los grupos más vulnerables socialmente. (L1; L2; L3; A2; A5; TD1).

La dimensión transformadora se observa cuando se incorpora este valor asociado al aprendizaje instrumental, es decir, cuando el aprendizaje se produce sobre la base de la colaboración para conseguir el éxito de todos y todas. Por el contrario, la dimensión exclusora, cuando se presenta el aprendizaje en términos competitivos y a nivel de mínimos.

5.7) Igualdad de diferencias: Sobre la base de la evidencia que la imposición de una igualdad homogeneizadora propicia la exclusión de los grupos minoritarios, al mismo tiempo que las reformas que favorecen la diversidad han fomentado desigualdades educativas. El aprendizaje dialógico se orienta hacia la igualdad de las diferencias afirmando que la verdadera igualdad respeta la diferencia, así, se asegura que todas las personas, desde su cultura, religión, género, estilo de vida, etc., tengan las mismas oportunidades a la vez que se fomenta la igualdad de resultados (éxito escolar, laboral y social). Por ello el currículum no está sesgado hacia ningún grupo, mientras que los objetivos educativos siguen siendo los mismos para todo el alumnado (L1; L2; L3; A2; A5).

La dimensión transformadora se evidencia en la búsqueda de la igualdad de resultados de aprendizaje sobre la base del respeto a la diferencia. Por el contrario, la dimensión exclusora, en el refuerzo de la diferencia y en la consecuente profundización de la desigualdad.

5.8) Emocionalidad: Se refiere al reconocimiento del otro desde su corporalidad hasta las relaciones y acciones sociales que establece con los demás, iniciándose en su reconocimiento en la emoción, entendido como aquella "capacidad para sentirse en plena empatía al estar en presencia del otro ... no hay posibilidad de tener cuerpo propio, a menos de que yo tenga la percepción del cuerpo del otro, hecho que sólo se puede interiorizar por esta capacidad empática y a su vez en la historización de mi cuerpo es fundamental la empatía con el otro"7. La negación del cuerpo en cuanto a esta forma de comprender la emocionalidad de cada niño y niña, básicamente por las historias personales de violencia que portan estos, muchas veces les impide iniciar proceso de interacción dialógico, colaborativo y eminentemente empático, pero no por ello no siguen siendo portadores de esta capacidad natural (A6).

La dimensión transformadora, de este principio, se evidencia en la aceptación de la corporalidad de cada persona en tanto ser amorosamente biológico que requiere ser reconocido como tal de forma previa y permanente a todo el proceso de aprendizaje. En cambio, la dimensión exclusora, obvia u omite esta característica básica de lo que significa el reconocimiento de lo humano.

Varela, F. (2000). La fenomenología de la vida. Santiago de Chile, Dolmen Ediciones. Varela plantea que al momento del nacimiento de un niño o niña "ver mover los brazos y mover los brazos es lo mismo" desde un punto de vista biológico, esto ejemplifica la génesis de la empatía humana (capacidad de ponerse en el lugar del otro) que se refuerza con la interacción social del sujeto en el medio social. Es decir, si un niño no desarrolla su emocionalidad empática en el espacio social en que se desarrolla porque está en un espacio de mucha violencia, por ejemplo, no quiere decir, que no porte esa capacidad, sino que hay que recuperarla en la acción social. 
El modelo de aprendizaje dialógico permite promover contextos de racionalidad comunicativa, en que se ejercita a cada momento la argumentación susceptible de crítica, conjuntamente con las relaciones de solidaridad. Al mismo tiempo, implica la posibilidad de poner en evidencia las pretensiones de poder que emergen durante los procesos de aprendizaje, con lo cual se profundizan los procesos reflexivos de carácter individual y colectivo.

6) MODELO DIDACTICO. Desde el marco conceptual de la pedagogía, una vez resueltas las cuestiones ¿qué enseñar? y ¿cómo lograr aprendizajes en el alumnado?, la pregunta ahora es ¿cómo enseñar lo seleccionado?

6.1. Metodología. En términos metodológicos, es posible construir un modelo didáctico de carácter eminentemente interactivo, que basa su accionar en las múltiples interacciones de la mayor diversidad de agentes sociales que sea posible ingresar al aula, a fin de ofrecer la mayor diversidad de formas de enseñar y formas de aprender. Esta metodología incorpora agentes sociales al trabajo pedagógico que se denominan "colaboradores de aprendizaje" que realizan un trabajo voluntario de colaboración en el aprendizaje, de preferencia al interior del aula pero también en otros espacios de aprendizaje en el centro escolar (como las bibliotecas o las aulas de informática), bajo la orientación del profesor responsable. Los colaboradores de aprendizaje son adultos provenientes de la comunidad cercana a la escuela, de la sociedad en general, o niños de los propios cursos, como también de cursos superiores de la propia escuela que manifiestan interés por colaborar con sus compañeros. Los colaboradores de aprendizaje, que constituyen un grupo muy heterogéneo a nivel cultural, lingüístico, religioso, educativo, de género, etc., se constituyen como un potente resorte pedagógico al interior del proceso transformador y mediatizan la multiplicidad de interacciones que se establecen en las relaciones pedagógicas dialogantes entre el alumnado, entre el alumnado y el profesorado, y entre el alumnado y los otros agentes sociales externos a la comunidad, a la vez que movilizan los aprendizajes instrumentales y sociales en el alumnado (L1; L2; L3; L4; A5; TD1; TD2; TD3; TD4; TM1; TM2; TM3; TM4; TL1).

La dimensión transformadora en esta categoría se observa en la incorporación de colaboradores de aprendizaje al trabajo pedagógico, con lo cual se ingresa directamente al aula a la comunidad social. En cambio, la dimensión exclusora, en la mantención de la comunidad social fuera del trabajo pedagógico.

6.2. Rol del profesorado. Radicalmente diferente al tradicionalmente asumido. Así, de ser un agente solitario y único moderador del proceso de enseñanza y aprendizaje, pasa a ser un coordinador profesional de un equipo de colaboradores de aprendizaje que ponen en práctica una diversidad de oportunidades de aprendizaje para el conjunto de sus estudiantes. De ser el único programador de las temáticas educativas, de las actividades y de las formas de evaluación, pasa a ser coordinador y negociador de las demandas pedagógicas de sus alumnos, de los apoderados y de la comunidad en general, para ser incorporadas al trabajo de aula y fuera de ella. De ser un informador y transmisor de la cultura del currículum obligatorio nacional, pasa a ser un constructor y resignificador del currículum. De ejercer un rol profesional circunscrito al trabajo de aula y la escuela, pasa a ser un investigador miembro de un equipo interdisciplinar que construye conocimiento 
científico y que luego transforma en conocimiento educativo. De ser un profesional cuyo ejercicio laboral se limitaba esencialmente al aula y la escuela, pasa a ser un difusor del conocimiento científico producido y del conocimiento educativo, más allá de su aula y más allá de su institución, participando activamente en eventos científicos, educativos y sociales (L1; L3; A5; L4; TD3; TM1, TM2; TM3; TM4; TL1).

La dimensión transformadora, en esta categoría, se observa en el cambio en las funciones profesionales del profesor, que ahora trabaja en conjunto con otros agentes sociales, donde su trabajo deja de ser privado y pasa a ser de dominio público. En cambio, la dimensión exclusora, en la mantención del rol profesional clásico, ejercicio profesional aislado y privado.

6.3. Rol del alumnado. En este modelo didáctico cambia radicalmente respecto de su rol tradicional. Así, de ser un agente pasivo receptor de conocimiento y de normativas establecidas, pasa a ser protagonista de la creación dialógica de conocimiento y negociador de nuevos acuerdos normativos más acordes con el requerimiento del nuevo contexto dialogizador que promueve el aula y la escuela en general. De tener un comportamiento de masa, pasa a tener un comportamiento de comunidad en que la colaboración constituye la esencia de las relaciones humanas. De estar circunscrito a las interacciones que le ofrecían el aula tradicional y la escuela, pasa a tener interacciones reales con un sinnúmero de agentes sociales que ingresan al aula (los colaboradores de aprendizajes). De estar categorizado por rangos de rendimiento académico y rotulado por diagnósticos clínicos, pasa a ser considerado en condiciones de igualdad con respeto de su propia diferencia. De ser considerado como un alumnado de bajas expectativas, a ser depositario de altas expectativas académicas y sociales (L1; L2; L3: L4; A6; TD3; TM1, TM2; TM3; TM4; TL1).

La dimensión transformadora se observa en el protagonismo asumido por parte del alumnado en cada una de las acciones que le involucran, así, la cooperación, la colaboración, la solidaridad y el trabajo comunitario se transforman en prácticas cotidianas. En cambio, la dimensión exclusora, el alumnado asume un comportamiento de acuerdo a rótulos, de carácter mayoritariamente pasivos.

6.4. Organización del aula. Cambia radicalmente de la tradicional. El aula se organiza, mayoritariamente, en lo que se conocen como grupos interactivos. Así, de trabajar con un aula organizada en filas de estudiantes, unos tras otros y que se mantienen fijos en sus puestos de trabajos, y donde sólo hay una persona adulta, se distribuye el alumnado en pequeños grupos, de número, dependiendo del tamaño del grupo-clase y de voluntarios en el aula, a cargo de un colaborador de aprendizaje, que rotan permanentemente, una vez finalizada cada actividad, para cambiarse al siguiente grupo, configurando un aula en permanente dinamismo. De trabajar en un aula que promueve eminentemente acciones educativas de carácter teleológico, a ser un aula que se desenvuelve en contextos de acciones comunicativas. De un trabajo pedagógico que utiliza el lenguaje sólo para alcanzar un objetivo, al uso de un lenguaje que permite actualizar y renovar consensos, transmitir y compartir emociones y sentimientos, así como proponerse alcanzar un entendimiento racionalmente motivado. De esta forma el proceso de enseñanza y aprendizaje se moviliza permanentemente en ambas direcciones, asumiendo el modelo de enseñante que aprende y aprendiente que enseña en cada una de la interacciones que 
mantiene durante el proceso formador. En la dinámica dialógica de grupos interactivos dos procesos ocurren al mismo tiempo: aumenta el aprendizaje instrumental porque se multiplican las actividades de enseñanza y aprendizaje para todos y todas, así como mejora la solidaridad de base al no excluir a ningún estudiante de los aprendizajes que son valorados en la actual sociedad del conocimiento (L1; L3; L4; A5; TM1; TM2; TM3; TM4; TL1).

Un ejemplo gráfico de la organización del grupo interactivo es el siguiente:

\begin{tabular}{|l|l|l|l|l|l|l|}
\hline \multicolumn{1}{|c|}{$\begin{array}{c}\text { Grupo } \\
\text { interactivo 1 }\end{array}$} & \multicolumn{1}{c|}{$\begin{array}{c}\text { Grupo } \\
\text { interactivo 2 }\end{array}$} & \multicolumn{1}{c|}{$\begin{array}{c}\text { Grupo } \\
\text { interactivo 3 }\end{array}$} & $\begin{array}{c}\text { Grupo } \\
\text { interactivo 4 }\end{array}$ & $\begin{array}{c}\text { Grupo } \\
\text { interactivo 5 }\end{array}$ & $\begin{array}{c}\text { Grupo } \\
\text { interactivo 6 }\end{array}$ & $\begin{array}{c}\text { Grupo } \\
\text { interactivo 7 }\end{array}$ \\
\hline $\begin{array}{l}\text { Colaborador } \\
\text { Académico }\end{array}$ & $\begin{array}{l}\text { Colaborador } \\
\text { Estudiante de } \\
\text { un curso su- } \\
\text { perior de la } \\
\text { escuela }\end{array}$ & $\begin{array}{l}\text { Colaborador } \\
\text { Apoderado }\end{array}$ & $\begin{array}{l}\text { Colaborador } \\
\text { Estudian te } \\
\text { universitario }\end{array}$ & $\begin{array}{l}\text { Colaborador } \\
\text { Profesional al } \\
\text { externo a la } \\
\text { escuela }\end{array}$ & $\begin{array}{l}\text { Colaborador } \\
\text { Estudiante par } \\
\text { del curso }\end{array}$ & $\begin{array}{l}\text { Colaborador } \\
\text { Estudiante de } \\
\text { postgrado }\end{array}$ \\
\hline Actividad 1 & Actividad 2 & Actividad 3 & Actividad 4 & Actividad 5 & Actividad 6 & Actividad 7 \\
\hline
\end{tabular}

En la distribución espacio temporal del aula con grupo interactivo cada grupo tiene una actividad que contempla un mismo contenido (instrumental y social), pero que da cuenta de una estrategia de aprendizaje diferente. La duración de la actividad es de un máximo de 20 minutos, luego cada grupo avanza a la actividad siguiente, en la cual tendrá una nueva oportunidad de aprendizaje con una persona adulta diferente, de tal forma que en un solo bloque toda la clase puede llegar a tener siete posibilidades distintas para aprender un mismo contenido instrumental y social.

La dimensión transformadora, en esta categoría, se observa desde una nueva distribución del alumnado, inclusiva y dialógica, hasta la atención personalizada para cada estudiante dando cuenta del estilo y ritmos de aprendizaje de cada uno y de distintos tipos de enseñanza en una misma clase, de los tiempos de concentración y de la heterogeneidad del alumnado. En cambio, la dimensión exclusora, en la mantención tradicional del aula, de un solo estilo de aprendizaje y enseñanza, con una sola persona adulta y de un solo perfil, de la homogeneidad y de la omisión de los tiempos de concentración.

En consecuencia, este modelo didáctico dialógico interactivo permite dar cuenta de 1) la vinculación entre el aprendizaje instrumental y el social al interior de cada actividad programada; 2) la diversificación de estrategias de aprendizaje en cada actividad programada, 3) dar cuenta de los períodos breves de atención del alumnado cuidando la duración de las actividades programadas; 4) considerar la heterogeneidad de los estudiantes, en cuanto a cultura, a género, a etnias, a edades, a rendimiento, a afinidades; 5) asegurar la atención personalizada, mediante la formación de grupos pequeños con un colaborador de aprendizaje cada uno; 6) dar cuenta de un grupo diversificado de agentes sociales que trabajan voluntariamente como colaboradores de aprendizaje y que diversificarán las interacciones del estudiantado, a la vez que, constituirán otros referentes pedagógicos distintos al profesor; 7) dar cuenta de un rol profesional que asume el profesor a través de la coordinación del trabajo pedagógico entre todos los colaboradores y todos sus estudiantes; 8) dar cuenta de un rol de estudiante protagónico, constructivo y colaborativo en su aprendizaje y en el de sus pares en forma permanente; 9) la generación permanente 
de altas expectativas en todo el alumnado de parte del profesorado, los colaboradores de aprendizaje y los familiares. Asimismo, esta metodología de trabajo de grupos interactivos garantiza la no segregación del alumnado, dada por la alta heterogeneidad con que se conforman los grupos y por su rotación permanente de un grupo a otro, y por la no separación del alumnado en función de atributos personales, tales como mayor o menor rendimiento académico, género, cultura, desarrollo cognitivo.

7) CONCEPTO DE EVALUACION. Se asume un concepto evaluativo dialógico, donde la característica principal, es que implica que la emisión de los juicios educativos debe realizarse sobre la base de los criterios posibles de ser objetivados, desde la perspectiva de las relaciones sociales que mantiene el alumnado con los distintos tipos de mundos a los que se enfrentan, tanto cuando deben aprender los saberes instrumentales, es decir, criterios de verdad y eficacia, como cuando deben aprender los saberes sociales, es decir, criterios de veracidad, rectitud y autenticidad. Estos criterios, a su vez, permiten dar cuenta de los ocho principios del aprendizaje dialógico. La evaluación se asume también en su dimensión instrumental, es decir, desde la calificación hasta la evaluación de resultados de aprendizaje de acuerdo a indicadores estandarizados; como en su dimensión social, es decir, evaluaciones comunicativas cualitativas que evidencian superación del conflicto en el aula, fortalecimiento de una cultura de colaboración, superación de violencia de género y étnicas. Las evaluaciones se realizan al interior de todos los grupos interactivos, con participación de los colaboradores de aprendizaje, así como también se realizan de parte solo del profesorado pero en un contexto en el que existen las estructuras para que la comunidad exprese su opinión al respecto y se pueda dialogar sobre ello (TD1; TD2; TM1; TM2; TM3; TM4; I1; I2).

La dimensión transformadora se evidencia en esta categoría en que el concepto de evaluación pierde su connotación punitiva para consolidarse en una connotación promovedora de los aprendizajes instrumentales y sociales dirigidos a los máximos para todo el alumnado, al mismo tiempo que fortalece la cultura de la colaboración entre pares y entre alumnado y colaboradores de aprendizaje. Por el contrario, la dimensión exclusora, asume el concepto de evaluación a fin de discriminar diferencias individuales fortaleciendo la competitividad y el carácter punitivo que tradicionalmente porta este concepto.

\section{SINTESIS DEL MODELO DIALOGICO DE LA PEDAGOGIA}

\begin{tabular}{|l|l|l|}
\hline \multicolumn{1}{|c|}{ CATEGORIA } & SUBCATEGORIA & \multicolumn{1}{c|}{ CONSTRUCCION CATEGORIAL } \\
\hline $\begin{array}{l}\text { CONCEPTO DE } \\
\text { EDUCACION }\end{array}$ & & $\begin{array}{l}\text { Medio para promover interacciones humanas dirigidas } \\
\text { a transformar las propias construcciones intersubjetivas } \\
\text { de quienes participan en el acto educativo al interior } \\
\text { de una comunidad con predominio de la racionalidad } \\
\text { comunicativa. }\end{array}$ \\
\hline $\begin{array}{l}\text { TIPO DE } \\
\text { CONSTRUCCION } \\
\text { DE LA PERSONA }\end{array}$ & & $\begin{array}{l}\text { La persona se construye sobre la base de núcleo } \\
\text { intersubjetivo-autopoiético. Esto opera como sustento } \\
\text { para la conducción de un proceso transformador que } \\
\text { requiere de la persona y de su propio colectivo en el } \\
\text { agenciamiento de sus propias intenciones educativas } \\
\text { y sociales, y de las de los demás. }\end{array}$ \\
\hline
\end{tabular}




\begin{tabular}{|c|c|c|}
\hline CATEGORIA & SUBCATEGORIA & CONSTRUCCION CATEGORIAL \\
\hline $\begin{array}{l}\text { TIPO DE } \\
\text { SOCIEDAD }\end{array}$ & & $\begin{array}{l}\text { Sociedades dialógicas, en que emerge como eje central } \\
\text { la necesidad del trabajo en redes solidarias, comunita- } \\
\text { rias y colaborativas entre los sujetos que las componen. } \\
\text { Esto es la base de un currículum que propone el trabajo } \\
\text { pedagógico de forma comunitaria. }\end{array}$ \\
\hline \multirow{2}{*}{$\begin{array}{l}\text { ENFOQUE } \\
\text { CURRICULAR }\end{array}$} & $\begin{array}{l}\text { CONCEPTO DE } \\
\text { CURRICULUM }\end{array}$ & $\begin{array}{l}\text { Construcción social que surge de las múltiples y } \\
\text { diversas interacciones de las personas que componen } \\
\text { una comunidad educativa contextualizada histórica y } \\
\text { socialmente, y que están sujetas tanto a relaciones de } \\
\text { imposición, como de diálogo. }\end{array}$ \\
\hline & $\begin{array}{l}\text { SELECCION DEL } \\
\text { CONOCIMIENTO } \\
\text { EDUCATIVO }\end{array}$ & $\begin{array}{l}\text { Los criterios de selección emanan de la propia } \\
\text { comunidad que decide el currículum, y los saberes } \\
\text { seleccionados consideran los propios requeridos por } \\
\text { el sistema, como de aquellos emergentes del mundo } \\
\text { de la vida. }\end{array}$ \\
\hline \multirow{8}{*}{$\begin{array}{l}\text { MODELO DE } \\
\text { APRENDIZAJE }\end{array}$} & $\begin{array}{l}\text { DIALOGO } \\
\text { IGUALITARIO }\end{array}$ & $\begin{array}{l}\text { Se requiere de una participación en un plano de igual- } \\
\text { dad, donde son importantes los aportes de cada uno de } \\
\text { los integrantes, según los argumentos presentados, no } \\
\text { según la mayoría o según una imposición de poder. }\end{array}$ \\
\hline & $\begin{array}{l}\text { INTELIGENCIA } \\
\text { CULTURAL }\end{array}$ & $\begin{array}{l}\text { Es la capacidad de aprender a través del diálogo y de } \\
\text { las habilidades comunicativas que tienen las personas. } \\
\text { Desde este concepto todos los estudiantes y toda la } \\
\text { comunidad son portadores de inteligencia. }\end{array}$ \\
\hline & TRANFORMACION & $\begin{array}{l}\text { Es la superación del recurso de dictamen como } \\
\text { etiquetaje paralizador y modificarlo en instrumen- } \\
\text { to de transformación de una situación personal o } \\
\text { social, enfatizando la transformación del contexto de } \\
\text { aprendizaje. }\end{array}$ \\
\hline & $\begin{array}{l}\text { DIMENSION } \\
\text { INSTRUMENTAL }\end{array}$ & $\begin{array}{l}\text { Son los conocimientos científicos y técnicos y el } \\
\text { desarrollo de habilidades instrumentales necesarios } \\
\text { para actuar con éxito en la sociedad. }\end{array}$ \\
\hline & $\begin{array}{l}\text { CREACION DE } \\
\text { SENTIDO }\end{array}$ & $\begin{array}{l}\text { Dotar al estudiantado de múltiples proyectos de vida } \\
\text { desde los cuales pueda tener alternativas de elección }\end{array}$ \\
\hline & SOLIDARIDAD & $\begin{array}{l}\text { Es un valor que se consigue sólo a través de la práctica } \\
\text { solidaria, como un valor que se pone en práctica en las } \\
\text { interacciones que desarrollamos en el aula y, en primer } \\
\text { lugar, por parte de las profesoras y profesores. }\end{array}$ \\
\hline & $\begin{array}{l}\text { IGUALDAD DE } \\
\text { DIFERENCIAS }\end{array}$ & $\begin{array}{l}\text { La verdadera igualdad respeta la diferencia, así, se } \\
\text { asegura que todas las personas tengan las mismas } \\
\text { oportunidades a la vez que se fomenta la igualdad de } \\
\text { resultados (éxito escolar, laboral y social). }\end{array}$ \\
\hline & EMOCIONALIDAD & $\begin{array}{l}\text { Es la aceptación de la corporalidad de cada persona } \\
\text { en tanto ser amorosamente biológico que requiere ser } \\
\text { reconocido como tal de forma previa al proceso de } \\
\text { aprendizaje escolar. }\end{array}$ \\
\hline
\end{tabular}




\begin{tabular}{|c|c|c|}
\hline CATEGORIA & SUBCATEGORIA & CONSTRUCCION CATEGORIAL \\
\hline \multirow{5}{*}{$\begin{array}{l}\text { MODELO } \\
\text { DIDACTICO }\end{array}$} & METODOLOGIA & $\begin{array}{l}\text { Incorpora agentes sociales al trabajo pedagógico que } \\
\text { se denominan "colaboradores de aprendizaje" que } \\
\text { realizan un trabajo voluntario de colaboración en el } \\
\text { aprendizaje, de preferencia al interior del aula, bajo } \\
\text { la orientación del profesor responsable. }\end{array}$ \\
\hline & $\begin{array}{l}\text { ROL DEL } \\
\text { PROFESORADO }\end{array}$ & $\begin{array}{l}\text { Nuevas funciones profesionales, ahora trabaja en con- } \\
\text { junto con otros agentes sociales, donde su trabajo deja } \\
\text { de estar situado en un aula privada para constituirse } \\
\text { en aula pública. }\end{array}$ \\
\hline & ROL DEL ALUMNADO & $\begin{array}{l}\text { Protagonismo asumido en la construcción intersubjeti- } \\
\text { va del aprendizaje por parte del alumnado en cada una } \\
\text { de las acciones que le involucran, así, la cooperación, } \\
\text { la colaboración, la solidaridad y el trabajo comunitario } \\
\text { se transforman en prácticas cotidianas. }\end{array}$ \\
\hline & $\begin{array}{l}\text { ORGANIZACION } \\
\text { DEL AULA }\end{array}$ & $\begin{array}{l}\text { Es inclusiva y se organiza en grupos interactivos, } \\
\text { pequeños grupos flexibles que rotan cada } 20 \text { min, } \\
\text { con una persona adulta en cada uno que dinamiza } \\
\text { las interacciones entre estudiantes diversos. Dando } \\
\text { cuenta de forma igualitaria de distintos estilos y } \\
\text { ritmos de aprendizajes y enseñanzas, períodos de } \\
\text { concentración. }\end{array}$ \\
\hline & $\begin{array}{l}\text { CONCEPTO DE } \\
\text { EVALUACION }\end{array}$ & $\begin{array}{l}\text { Medio para promover los aprendizajes instrumentales } \\
\text { y sociales dirigidos a los máximos para todo el alum- } \\
\text { nado, al mismo tiempo que fortalece la cultura de la } \\
\text { colaboración entre pares y entre alumnado y colabo- } \\
\text { radores de aprendizaje. Evaluación de aprendizajes } \\
\text { instrumentales y sociales. Dimensiones cuantitativas } \\
\text { y cualitativas del aprendizaje. }\end{array}$ \\
\hline
\end{tabular}

\section{CONCLUSIONES}

El análisis de contenidos de texto realizado a las unidades de estudio seleccionadas permite dar cuenta de un modelo pedagógico que se reconstruye desde los planos teóricos y operativos, definidos en el presente artículo:

1. La pedagogía dialógica desde el plano teórico. El modelo dialógico de la pedagogía orienta toda su acción en la adopción de un concepto de educación entendido como un medio para promover interacciones humanas dirigidas a transformar las propias construcciones intersubjetivas de quienes participan en el acto educativo al interior de una comunidad con predominio de la racionalidad comunicativa. Todo lo cual, avalado y en total correspondencia con la explicación de que la formación de la persona obedece eminentemente a un proceso intersubjetivo con base biológica y social, en que las personas de referencia resultan fundamentales en la construcción de la misma. Desde 
este planteamiento, el concepto de educación puede sostener la transformación de esas construcciones intersubjetivas en sus procesos pedagógicos. De esta forma, se asume que la persona es capaz de agenciar cambios al interactuar con otras. Por consiguiente, la respuesta a la pregunta sobre ¿qué tipo de persona se desea formar? se puede sintetizar en una persona con altas capacidades de interacción social y para hacer frente a los retos de la sociedad del conocimiento, dialógica, solidaria, comprometida con la transformación del medio en que se desenvuelve.

Ahora bien, estos antecedentes sólo adquieren sentido al responder la otra pregunta que cierra este plano teórico que asume la pedagogía: ¿qué tipo de sociedad se quiere construir? Se acepta que la sociedad funciona como sistema y mundo de la vida, lo cual requiere del desarrollo de redes solidarias, comunitarias y colaborativas entre los sujetos que las componen, en tanto es función de la pedagogía contribuir al desarrollo y fortalecimiento de estas redes

2. La pedagogía dialógica desde el plano operativo. En directa relación con lo asumido en el plano teórico, el ¿qué enseñar? queda resuelto en la incorporación de todos los saberes instrumentales y sociales que se consideran más fundamentales para la inclusión del alumnado en las sociedades actuales y sobre la base del acuerdo de la propia comunidad. Con lo cual se garantiza tanto la intersubjetividad resultado de la participación protagónica de todos los agentes involucrados, como la diversidad de interacciones que permite asegurar la transformación de las construcciones intersubjetivas de quienes participan. Al mismo tiempo, que se da cuenta de una formación tanto para responder al sistema como al mundo de la vida.

Estos saberes que constituyen el currículum entran en correspondencia con la explicación biológica y social del modelo dialógico del aprendizaje que da cuenta a través de sus ocho principios de cada uno de los elementos presentes en el plano teórico de la pedagogía. A su vez, este modelo encuentra expresión plena en el modelo didáctico interactivo asumido por este modelo pedagógico, en que se asegura tanto la interacción, la solidaridad, la colaboración, la dialogicidad en cada una de sus acciones pedagógicas. Para finalmente evaluar el proceso de aprendizaje del mismo desde un concepto que se hace cargo de los criterios evaluativos instrumentales (provenientes del sistema) como de los criterios sociales (provenientes del mundo de la vida) desde los cuales legitimar en última instancia la selección de los conocimientos educativos.

En consecuencia, los resultados de este estudio permiten evidenciar que el modelo dialógico de la pedagogía asienta todo su trabajo en la aceptación de que es posible transformar la realidad de la escuela vulnerable, pero se requiere para ello de: una intervención social en que la participación de la comunidad resulta determinante; la aceptación del principio de generación de altas expectativas para todo su alumnado, y de la generación de modelos pedagógicos distintos a los tradicionales.

\section{BIBLIOGRAFIA}

Aubert, A., M. Soler (2007). Dialogism: The diagotic turn in the social sciences. The Praeger Handbook of Educational and Psychology Vol. (3), Chapter 62, pp. 521-529.

Bernstein, B. (1988). Clase, código y control. II. Hacia una teoría de las transmisiones educativas, Madrid: Akal. 
Bernstein, B. (1993). La estructura del discurso pedagógico. Madrid: Morata.

Eisner, E. (2002a). Procesos cognitivos y currículum. Buenos Aires, Martínez Roca.

Eisner, E. (2002b). Educar la visión artística. Madrid: Paidós.

Eisner, E. (1998). Cognición y currículum, Buenos Aires: Amorrortu.

Eisner, E., E. Wallance (1974). Conflicting conceptions of curriculum. Berkeley, McCutchan Publishing Company.

Elboj, C., I. Puigdellívol, M. Soler, R. Valls (2002). Comunidades de aprendizaje. Transformar la educación. Barcelona: Graó.

Chevalard, Y. (1991). La transposición didáctica. Madrid: Aique.

Molero, F. (1999). La didáctica ante el tercer milenio. Madrid: Síntesis.

Ferrada, D. (2007). El principio de emocionalidad: Un aporte al modelo dialógico del aprendizaje. Revista REXE, Vol. 12 7: 22-36.

Flecha, R. (2000). Sharing Words. Lanham, M.D: Rowman \& Littlefield.

Gómez, J., A. Latorre, M. Sánchez, R. Flecha (2006). Metodología comunicativa crítica. Barcelona: El Roure.

Grundy, S. (1991). Producto o praxis del currículum. Madrid: Morata.

Hoyos, A. (1999). Epistemología y discurso pedagógico: razón y aporía en el proyecto de modernidad. En: Hoyos, A. (ed.). Epistemología y objeto pedagógico. ¿Es la pedagogía una ciencia? México: UNAM.

Labarrere, A., M. Quintanilla (2002). La solución de problemas científicos en el aula. Reflexiones desde los planos de análisis y desarrollo. Revista Pensamiento Educativo, Vol. 30: 121-138.

Lundgren, U. P. (1992). Teoría del currículum y escolarización. Madrid: Morata.

Meneses, G. (1999). Epistemología y pedagogía. En: Hoyos, A. (ed.). Epistemología y objeto pedagógico. ¿Es la pedagogía una ciencia? México, UNAM.

Porlan, R., A. Rivero (1998). El conocimiento de los profesores. Sevilla: Diada Editora.

Pozo, I., M. Gómez (2003). Aprender y enseñar ciencia. Madrid: Morata.

Quintanilla, M., M. Izquierdo, A. Adúriz-Bravo (2005). Characteristics and methodological discusion about a theoretical model that introduces the history of science at an early stage of experimental science teachers' professional formation. Proceeding of IHST International Conference of history and philosophy of science and science teaching, University of Leeds.

Racionero, S., R. Valls (2007). Dialogic Learning: A communicative approach to teaching and learning. The Praeger Handbook of Educational and Psychology, Vol. (3), Chapter 65: 548557.

Sánchez, M. (1999). La Verneda-Sant Martí: A School where people dare to dream. Harvard Educational Review, Vol. 69, 3: 2-24.

Soto, V. (1996). Teorías críticas y su impacto en la construcción del currículum. Santiago de Chile, UMCE.

Varela, F. (2000). La fenomenología de la vida. Santiago de Chile: Dolmen Ediciones. 
